\title{
The Oxidation Kinetics of Thin Copper Films Studied by Resistivity Measurements
}

\author{
M. Rauh, H.-U. Finzel ${ }^{\mathrm{a}}$, and P. Wißmann \\ Institute of Physical and Theoretical Chemistry of the University Erlangen-Nürnberg, \\ Egerlandstr. 3, D-91058 Erlangen \\ ${ }^{a}$ Fachhochschule Niederrhein, FB Physikalische Chemie, Adlerstr. 32, D-47998 Krefeld
}

Z. Naturforsch. 54 a, 117-123 (1999); received July 29, 1998

Resistivity measurements on thin metal films allow to study the kinetics of oxidation. The method is applied to $50-60 \mathrm{~nm}$ thick copper films deposited on glass substrates under UHV conditions. After annealing at $150^{\circ} \mathrm{C}$, the films are exposed to pure oxygen at various temperatures in the range $85-135^{\circ} \mathrm{C}$, and the electrical resistivity is recorded in situ. At these temperatures, the oxygen begins to penetrate into the interior of the films, which results in a relatively steep increase in the film resistivity. A linear time law is valid to good approximation, which can be attributed to the influence of the dissociation of an adsorbed molecular species of oxygen on the reaction velocity. A potential diffusion of oxygen in the grain boundaries is also discussed.

Key words: Oxidation; Kinetics; Resistivity; Thin Metal Films.

\section{Introduction}

The electrical resistivity of thin copper films increases during the interaction with oxygen [1 - 4]. The increase amounts to a few per cent at room temperature for a $50 \mathrm{~nm}$ thick film and hence is typical for pure adsorption phenomena [5 - 7]. At elevated temperatures, however, the resistivity increase is enhanced by several orders of magnitude, which points to an absorption effect, i. e. the oxygen penetrates into the interior of the films. Similar phenomena were reported for the interaction of iron films with carbon [8] and palladium films with hydrogen [9].

The change in resistivity with time can easily be recorded time-resolved with high accuracy. Attempts of a quantitative interpretation of the absorption effect, however, are rare in the literature [10 - 12]. The present work tries to fill this gap. The $\mathrm{Cu} / \mathrm{O}_{2}$ system serves as a model system because there exist extensive resistivity data on the adsorption of oxygen on thin copper films [1,3]. In addition, the oxidation kinetics has been investigated by optical measurements on the same films using ellipsometry [13].

\section{Reprint requests to Prof. P. Wißmann;}

Fax: +49 91318528867 .

\section{Experimental}

The experimental arrangement and the film structure have extensively been described in [1 - 3]. The copper films with thicknesses from 50 to $60 \mathrm{~nm}$ were deposited on glass substrates under ultrahigh-vacuum conditions. After annealing the films at $150{ }^{\circ} \mathrm{C}$ for $75 \mathrm{~min}$, they were exposed to oxygen at temperatures in the range 85 to $135^{\circ} \mathrm{C}$. The change in the resistivity with time was recorded in situ. After completion of the UHV experiments, the copper was chemically dissolved, and the initial copper thickness $d_{0}$ was determined using quantitative atomic absorption spectroscopy.

The structure of the pure and oxidized films has been investigated in previous work [1, 3, 14 - 15]. The polycrystalline films exhibit a (111) fiber texture, i. e. the (111) planes are preferably oriented parallel to the glass surface with no azimuthal alignment[14]. Scanning tunneling micrographs (STM) did show [15] that a certain roughness of the surface is still present after annealing.

\section{Evaluation of Resistivity Data}

The evaluation of the resistivity data is performed on the basis of the layer model [1] shown in Figure 1a. Obviously, the thickness of the metallic copper is 

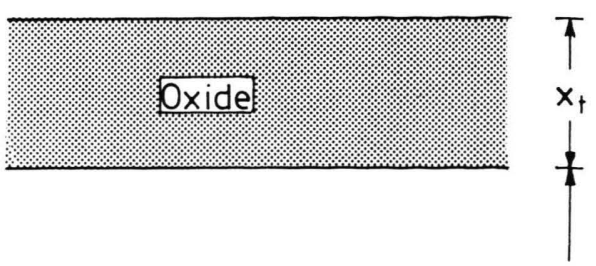

Copper

$d_{t}$

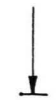

Fig. 1a. Layer model for the oxide formation.

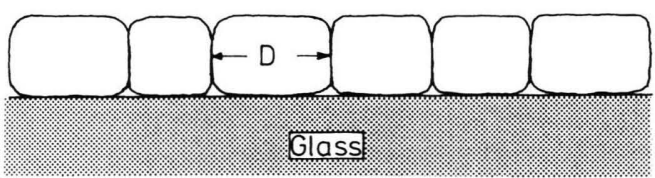

Fig. 1b. Schematic representation of the microstructure of the copper films annealed at $150{ }^{\circ} \mathrm{C}$.

reduced during oxidation. Further, we assume that the conductivity of the oxide layer is negligibly small as compared to that of the copper, and that the increase of resistivity during the oxidation process is exclusively due to the decreasing thickness of the copper film. The thickness of the growing oxide layer $X_{t}$ is then related to the copper thickness $d_{t}$ at the time $t$ by

$$
X_{t}=V\left(d_{0}-d_{t}\right)
$$

where $V$ is a factor describing the enhancement of the film volume during oxidation. For $\mathrm{Cu}_{2} \mathrm{O}$ as well as for $\mathrm{CuO} V \approx 1.7$ holds to a good approximation [13].

Since $d_{t}$ decreases with time, the measured resistance $R_{t}$ should increase according to

$$
R_{t} d_{t}=f \rho_{\mathrm{F}},
$$

where $f$ is a dimensionless geometric factor [3] and $\rho_{\mathrm{F}}$ the copper film resistivity, which should correspond to bulk copper samples with the same lattice defects as the copper films. It should be emphasized that $\rho_{\mathrm{F}}$ is assumed to be independent of the film thickness, contrary to the usual assumption [4]. Reasons for this constancy of $\rho_{\mathrm{F}}$ may be seen in the fact that the lattice disorder inside the copper films remains unchanged during oxidation. Moreover, surface scattering is rather small and can be neglected to a first approximation, as the example of oxygen adsorption on a $55 \mathrm{~nm}$ thick copper films at room temperature

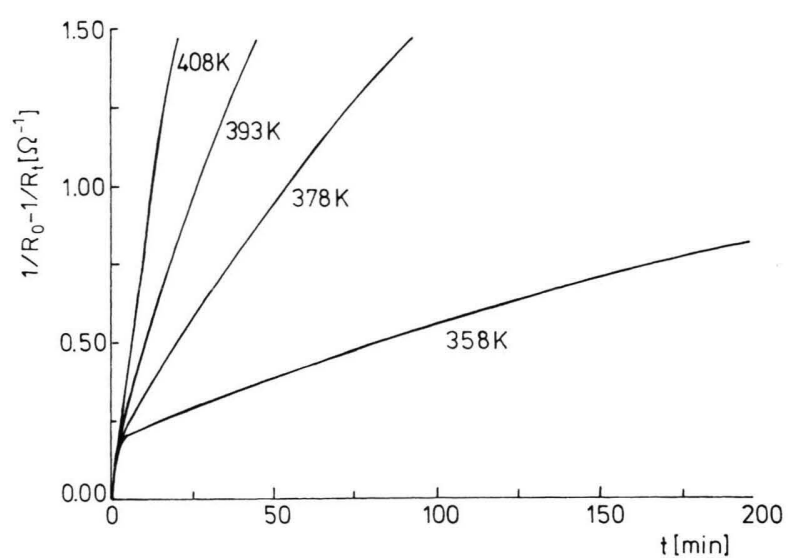

Fig. 2. Temperature dependence of the conductivity versus time curves during oxidation. $P_{\mathrm{O} 2}=31 \mathrm{~Pa}, d=60 \mathrm{~nm}$. The accuracy of the conductivity measurement is better than $1 \%$.

shows: a relative resistivity increase of $3.5 \%$ is observed [3], which indeed can be ignored in comparison with the drastic changes due to oxidation, at least for sufficiently high thicknesses of the copper films. It should be mentioned in this context that less than $45 \%$ of the initial copper thickness is oxidized in all experiments of Figure 2.

If a characteristic constant $C$ is defined according to

$$
C=V f \rho_{\mathrm{F}}
$$

we obtain from (1) - (3)

$$
X_{t}=C\left(R_{0}^{-1}-R_{t}^{-1}\right) .
$$

Obviously, it is advisable to discuss the dependence of the quantity $\left(R_{0}^{-1}-R_{t}^{-1}\right)$ on time in order to obtain information on the reaction mechanism. From literature we know that a linear time law

$$
X_{t}=k_{1} t
$$

is expected for phase boundary reactions dominating the oxidation kinetics. Such a behaviour has been observed, for example, for porous oxide layers [16]. If the oxide layer is homogeneous and strongly adhesive, then a parabolic time law

$$
X_{t}=k_{2} t^{1 / 2}
$$

should hold as reported by many groups [16 - 20]. 


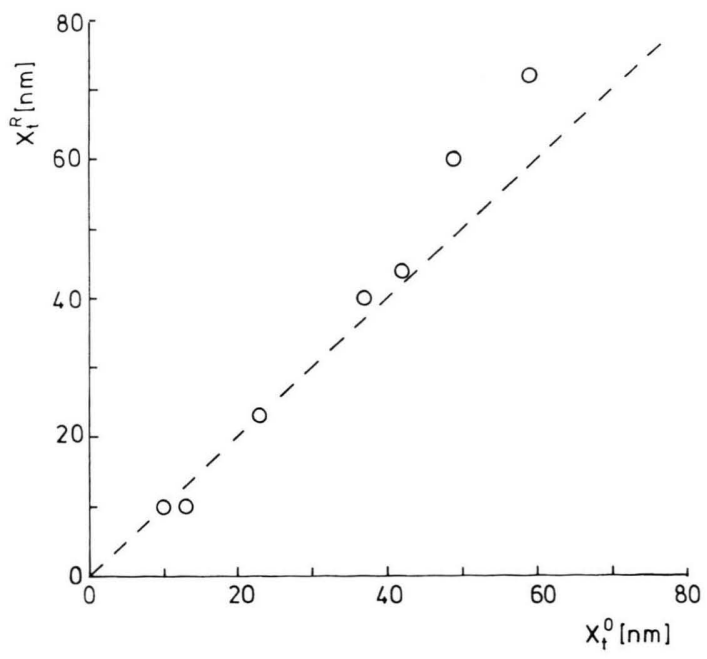

Fig. 3. Correlation of the oxide layer thickness, calculated on the basis of (4) $\left(X_{t}^{R}\right)$ with the ellipsometrically determined thickness $\left(X_{t}^{0}\right)$. The straight line has the slope -1 .

\section{Results and Discussion}

Typical conductivity versus time curves measured for various temperatures are shown in Figure 2. The thickness $d_{0}$ of the pure copper films was $60 \mathrm{~nm}$, the oxygen pressure in the gas phase $31 \mathrm{~Pa}$. Under these relatively mild conditions the oxygen starts with pure adsorption at the copper surface (initial increase of the curves in Figure 2). After reaching the monolayer coverage, the oxygen begins to penetrate into the interior of the film [1 - 3]. A more or less linear increase with time is found, where the slope strongly depends on temperature. Such a linear time law may be traced back to the dissociation of molecularly adsorbed oxygen as the rate determining step in the reaction [13], an interpretation well supported by the literature [21 - 22]. A parabolic law would be expected for a volume diffusion or a cation vacancy diffusion mechanism.

A linear time law was found in a wide range of time also in optical measurements on the same films [13]. From the dependence of $k_{1}$ in (5) on the temperature an activation energy of $64 \mathrm{~kJ}^{\text {mole }}{ }^{-1}$ was derived, in very good agreement with the present resistivity results.

Contrary to the proposal of Case [12], we have assumed that the oxide layer is completely insulating. This assumption seems to be justified, as resistivity measurements on oxidized films show. Total oxidation causes an enhancement of the film resistance by more than four orders of magnitude. As a consequence, the agreement between the thickness $X_{t}^{R}$ of the oxide layer calculated from (4) and the thickness $X_{t}^{0}$ derived from ellipsometrical measurements [13] is surprisingly good, at least for sufficiently small oxidation times (Figure 3 ). For rather high oxidation times the $X_{t}^{R}$ values are slightly larger. This deviation may be traced back to an increasing film roughness which influences the resistivity more markedly than the optical data [4].

Note that a slight curvature is detectable in all curves in Figure 2. Evidently, the effect of surface scattering is too small to explain this curvature. More likely the volume diffusion of oxygen as a rate determining step cannot be totally neglected.

\section{TCR Measurements}

Further hints to details of the oxidation process are provided by measurements of the temperature coefficient of resistivity (TCR). If the oxidation only reduces the cross section effective for the metallic conduction, the temperature coefficient $\alpha$, defined by

$$
\alpha=\frac{1}{\rho} \frac{\mathrm{d} \rho}{\mathrm{d} T}
$$

should remain constant [6].

The experimental data are shown in Figure 4. The TCR is plotted versus a normalized oxide thickness according to (4). Indeed, $\alpha$ does not change much with the oxide thickness over a wide thickness range. Only

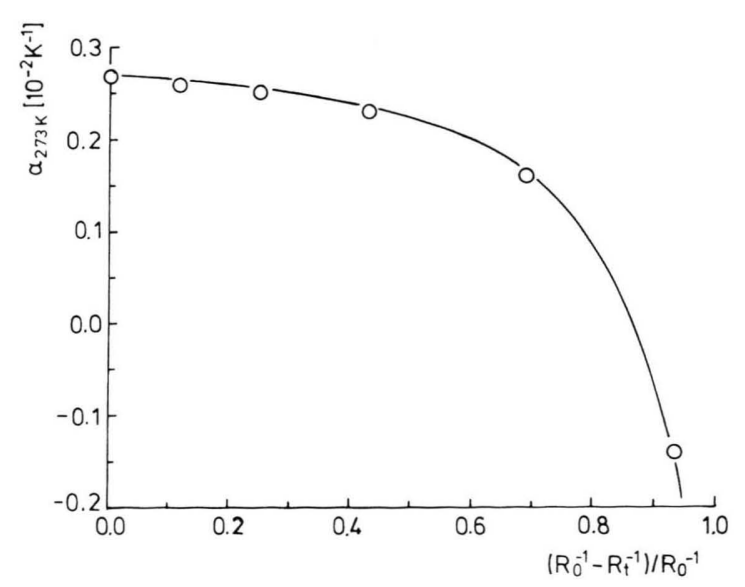

Fig. 4. Change of the TCR (alpha) of the film of Fig. 2 $\left(T=105^{\circ} \mathrm{C}\right.$ ) with oxidation progress (refer to (4) and (7), respectively). 


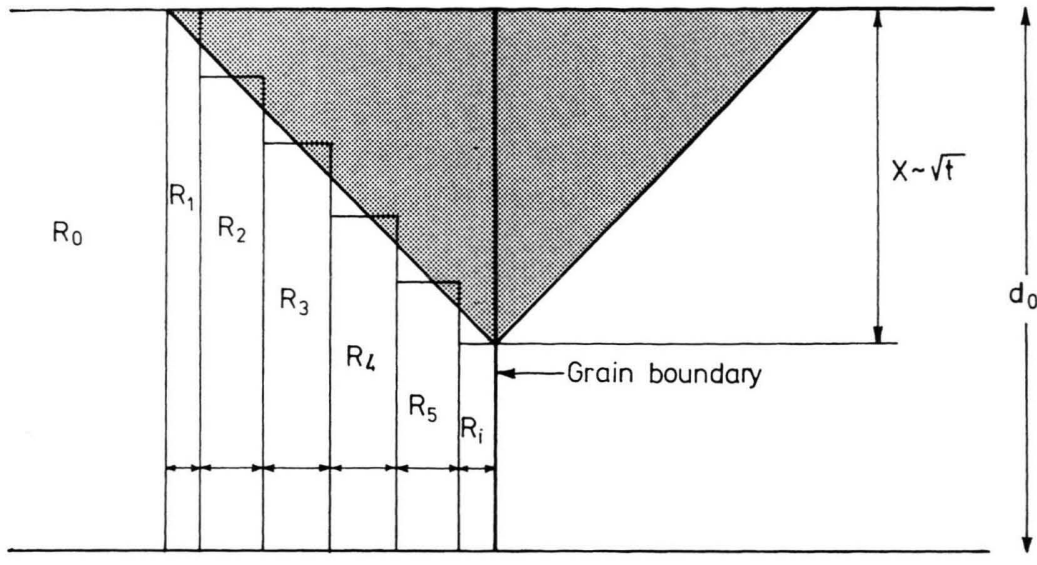

Fig. 5. Schematic representation of the model used for the calculation of the film resistivity.

for large oxide thicknesses, $\alpha$ decreases and even becomes negative, which is expected if the conduction electrons have to pass through oxidized and hence semiconducting film portions.

\section{Oxygen Diffusion in the Grain Boundaries}

Up to now we have restricted ourselves to the discussion of the simple layer model shown in Fig. 1a, and we have totally neglected the influence of the real microstructure of the films. However, x-ray diffraction [14], electron microscopy [3] and scanning tunneling microscopy investigations [15] have led to the conclusion that the schematic picture of Fig. $1 \mathrm{~b}$ is closer to reality in the present case. The films consist of small crystallites separated from each other by grain boundaries. The extension $D$ of the crystallites in the film plane is slightly larger than the extension in the direction perpendicular to the film plane [6].

For such films, an influence of the grain boundary diffusion of oxygen on the oxidation kinetics must be discussed [16, 23], taking into account that the grain boundaries in the films contain a certain amount of residual gas included during deposition [6]. Hence the grain boundaries in the films are much more distorted than grain boundaries in bulk copper [24], which favours the penetration of oxygen in these "easy paths of diffusion".

We have performed calculations of the effect of grain boundaries on the electrical resitivity in order to demonstrate alternative possibilities of the interpretation of a linear time law for the oxidation. The proposed model is based on very rough assumptions and by no means a realistic description of the grain boundary diffusion. Many restrictions remain ambiguous in the calculation and need further clarification. Amongst others, we should mention:

(1) A linear model of the grain boundary is applied in spite of the fact that, strictly spoken, a twodimensional approach is necessary in order to describe the inhomogeneities in the current distribution. A certain justification for a one-dimensional treatment may be seen in the calculations of the grain boundary scattering [25] and roughness effects [26 - 27] where reasonable results have been reported.

(2) Probably, a uniform phase of $\mathrm{Cu}_{2} \mathrm{O}$ is formed in the insulating domains $[1,14]$. A monoclinic oxygenrich $\mathrm{CuO}$ phase, however, is also discussed in the literature [17, 28, 29], at least in a near-surface region [30]. Since the physical characteristics of such a surface layer cannot yet be specified, we have totally omitted the effect of a change of the oxygen stoichiometry inside the oxide layer.

(3) The volume oxidation $[18,31]$ has been neglected as compared to the grain boundary diffusion of the oxygen. This may be the most important reason for the small curvature of the curves in Figure 2. Nevertheless, the grain boundary density is extremely high in the films ( $D$ in Fig. 1b is of the same order of magnitude as the film thickness $d_{0}$ ).

(4) A triangle shape of the oxide formed in the grain boundaries has been proposed [32]. The structure of the grain boundary, however, needs further specification with the help of transmission electron micrographs in future work. Some support for the triangle shape may be seen in an exact solution of the diffusion equation [33] which leads to a profile of oxygen concentration quite similar to that proposed by Shewmon [32]. A further argument for the triangle shape is based on the fact that the activation energy 


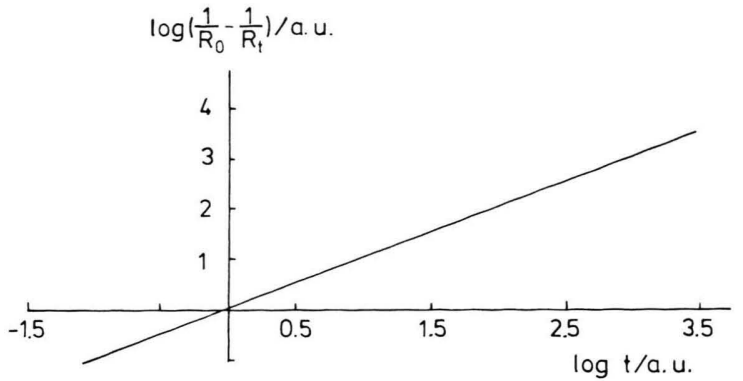

Fig. 6. Calculated conductivity versus time curves in a plot like in Fig. 2 but on a doubly logarithmic scale. For details see text.

for the oxygen diffusion in the (111)-direction of the net planes [31] as well as in the grain boundaries [16] both are distinctly smaller than the activation energy for bulk copper oxidation mentioned above.

(5) The bottom peak of the triangle in Fig. 5 (angle of inclination $45^{\circ}$ ) is assumed to move along the grain boundary according to a parabolic time law. This assumption is based on the idea that the grain boundary can be described by "smeared vacancies" [35] and hence has properties of an inner surface where a hopping mechanism is expected for the diffusion of adsorbed gases [36]. Note, however, that the grain boundaries can be blocked by the oxygen itself which mathematically results in a time dependence of the diffusion coefficient and hence in a deviation from the parabolic time law $[34,36]$.

(6) The theoretical curve of Fig. 6 was calculated only up to a maximum oxidation depth which corresponds to a remaining copper thickness under the bottom peak of the triangle in Fig. 5 of $0.4 d_{0}$, or to $0.6 l_{0}$ where $l_{0}=38 \mathrm{~nm}$ [37] is the mean free path of the conduction electrons in copper. By this restriction we intended to reduce a possible contribution of a low-dimensionality of the remaining copper layer [7] or even a tunneling of electrons through the triangle tip on the resistivity.

(7) The copper crystallites are enlarged in the direction of the film plane during annealing [15]. Hence the distance between two grain boundaries was set equal to $D=70 \mathrm{~nm}$ for a $55 \mathrm{~nm}$ thick film. In a description closer to reality, however, the scattering of the $D$ values around an average should be taken into account [4].

(8) In the framework of the model shown in Fig. 5 , scattering centers at the copper/oxide interface in the grain boundaries induce a microscopic roughness with a periodicity and asperity height small againt $l_{0}$, and of the same order of magnitude as the Fermi wavelength of the electrons. Such a roughness can be described by suitable p-factors [38 - 39] and will thus lead to only minor corrections of the model presented above.

(9) The oxidation of the grain boundaries can be incomplete, and channels of high conductivity through the grain boundaries may alter the current distribution seriously. A few percolation paths in the oxide can result in a time dependence strongly differing from the simple model. Even a cubic [40] or a logarithmic time law [29] was reported in this context.

The situation shown in Fig. 5 may serve as a first approach to the interaction with oxygen after the time interval $t$. The oxide area (shaded) is embedded into the remaining copper film (bare). The total resistivity of the films including the grain boundary can be easily calculated by classical network analysis. The layers are devided in perpendicular segments denoted in Fig. 5 by $R_{0}, R_{1}, R_{2}$ etc. By adding all resistances in series (each segment consists of an oxide and a metal portion in parallel) we obtain the total resistivity in relative units. The ratio of the resistivities of copper and copper oxide was set equal to 10000 because of the measurements on totally oxidized films mentioned in Section 4. The step height of the wall edges of the triangle is equidistantly set to $0.2 \mathrm{~nm}$ which approximately corresponds to the distance of two (111) oriented net planes in copper.

Figure 6 shows the result of the calculation. In order to make possible a comparison with (4) we have plotted $\left(1 / R_{0}-1 / R_{t}\right)$ versus time $t$, both on logarithmic scales. A direct comparison with the data of Fig. 2 is not recommendable because of the initial adsorption effect, the curvature of the curves of Fig. 2, and the unknown constant $k_{1}$ in (5). Nevertheless, the simple model offers a theoretical time dependence in spite of the fact that simplicity does not guarantee a realistic physical background even if the experimental facts are described reasonably well.

One easily recognizes that the theoretical curves can be approximated by straight lines with the slope -1 . Hence we can generally state that the diffusion of oxygen in the grain boundaries causes the time law to shift from a parabolic to a linear type, similar to a phase boundary reaction as a rate determining step. Future work is necessary to clear up the real importance of grain boundaries for the oxidation kinetics of the highly distorted films discussed here. 


\section{Conclusions}

The oxidation kinetics of thin copper films was studied by resistivity measurements. A nearly linear time law was found, which points to oxygen dissociation at the surface to be the rate determining step. Model calculations show, however, that a preferred diffusion of oxygen in the grain boundaries can also explain the experimental data. Even the ellipsometrical data of Rauh et al. [13] are in agreement with such an interpretation. Hence a complete structural characterization of the oxidized grain boundaries is necessary in order to make a decision between the various mechanisms for the interpretation of the experimental data. Another possibility to get more insight into the physics of oxidation kinetics is to perform resistivity measurements on single-crystal copper films. Such films can be easily prepared by depositing on clean $\mathrm{Si}(111)$ substrates which should be highly ohmic in order to prevent a short-circuiting of the film [6]. Since single-crystal films do not contain large-angle grain boundaries, the oxidation rate

[1] P. Wißmann in: Growth and Applications of Thin Films (eds. L. Eckertova and T. Ruzicka), Prometheus Publ. Prague 1994, p. 25.

[2] R. Schmidt and P. Wißmann, Surf. Interface Anal. 12, 407 (1988).

[3] H. Buck, R. Schmidt, and P. Wißmann, in Nichtmetalle in Metallen, ed. by D. Hirschfeld, Verlag DGMInformationsgesellschaft, Wiesbaden 1988, pp. 219.

[4] D. Dayal, H.-U. Finzel, and P. Wißmann, in Thin Metal Films and Gas Chemisorption, ed. by P. Wißmann, Elsevier Publ. Amsterdam 1987, pp. 53.

[5] J. W. Geus in Chemisorption and Reactions on Metallic Films Vol 1, ed. by J. R. Anderson, Academic Press London 1971, pp. 327.

[6] P. Wißmann, in Springer Tracts in Modern Physics Vol. 77, ed. by G. Höhler, Springer-Verlag Berlin 1975, pp. 1.

[7] D. Schumacher, Surface Scattering Experiments with Conduction Electrons, Springer Tracts Modern Physics Vol. 128 (1993).

[8] G. Wedler, in Thin Metal Films and Gas Chemisorption, ed. by P. Wißmann, Elsevier Publ. Amsterdam 1987, pp. 433.

[9] P. Wißmann, G. Wedler, and M. Watanabe, in Nichtmetalle in Metallen, ed. by D. Hirschfeld, Verlag DGMInformationsgesellschaft, Wiesbaden 1990, pp. 49. should be drastically lowered if grain boundary diffusion is the rate-determining step in polycrystalline films. We plan corresponding measurements in future work.

The thickness of the oxide layer derived from the resistivity data correlates satisfactorily with that obtained from ellipsometric results on the same films in the case of moderate oxide thicknesses. Due to the increasing roughness, the thickness obtained from the resistivity data for thick copper oxide layers is too high. While comparing with the data of other groups, one should always keep in mind that excellent vacuum conditions are a prerequisite for reliable results [41]. Contaminations during film deposition often create additional paths of diffusion which may alter the kinetics of film oxidation in an unexpected manner. Moreover, the temperature is an important factor. In this context it should be mentioned that a parabolic time law was observed for copper films oxidized at temperatures still higher than reported here [2].

Thanks are due to the Fonds der Chemischen Industrie for a generous financial support of this work.

[10] S. Stotz, Ber. Bunsengeses. Phys. Chem. 70, 769 (1966)..

[11] I. M. Ritchie, in Chemisorption and Reactions on Metallic Films, Vol. 2, ed. by J. A. Anderson, Academic Press, London 1971, pp. 257.

[12] F. C. Case, J. Vac. Sci. Technol A6, 123 (1988).

[13] M. Rauh and P. Wißmann, Thin Solid Films 228, 121 (1993).

[14] R. Gebhardt, A. Krehan, M. Rauh, and P. Wißmann, Fresenius Z. Anal. Chem. 341, 332 (1991).

[15] P. Wißmann, in Handbook of Optical Properties, Vol. II, Optics of small Particles, Interfaces, and Surfaces, ed. by R.E. Hummel and P. Wißmann, CRC Publ., Boca Raton 1997, pp. 401.

[16] G. Hörz, in Gase und Kohlenstoff in Metallen, ed. by E. Fromm and E. Gebhardt, Springer-Verlag, Berlin 1976, pp. 84.

[17] H. Neumeister and W. Jaenicke, Z. Phys. Chem. (Frankfurt) 108, 217 (1977).

[18] K. Hauffe, Reaktionen in und an festen Stoffen, Springer-Verlag, Berlin (1966).

[19] C. Wagner, Ber. Bunsenges. Phys. Chem. 70, 775 (1966).

[20] R. E. Hummel, Phys. Stat. Sol. (a) 76, 11 (1983).

[21] K. Hardel and D. Schön, Z. Phys. Chem. (Frankfurt) 77, 293 (1972). 
[22] I. M. Ritchie and G. L. Hunt, Surface Sci. 15, 524 (1969).

[23] P. Wißmann and H. Zitzmann, Fresenius Z. Anal. Chem. 319, 591 (1984).

[24] T. Surholt, Y. M. Mishin, and C. Herzig, Phys. Rev. B 50, 3577 (1994).

[25] A. F. Mayadas and F. Shatzkes, Phys. Rev. B 1, 1382 (1970).

[26] Y. Namba, Japan. J. Appl. Phys. 9, 1326 (1970).

[27] H.-U. Finzel and P. Wißmann, Ann. Phys. 43, 5 (1986).

[28] L. Paoletti, P. A. Rosa, P. Picozzi, and S. Santucci, J. Vac. Sci. Technol. A 2, 1471 (1984).

[29] G. A. Adegboyega, Nuovo Cimento D 11, 969 (1989).

[30] M. Wu and P. J. Möller, Phys. Rev. B 40, 6063 (1989).

[31] J. Benard, F. Grönlund, J. Oudar, and M. Duret, Z. Elektrochem. 63, 799 (1959).

[32] P. G. Shewmon in Physical Metallurgy, ed. by R. W. Cahn, North-Holland Publ. Amsterdam 1970, p. 397.
[33] I. Kaur and W. Gust, Fundamentals of Grain and Interphase Boundary Diffusion, Ziegler Press, Stuttgart 1988, pp. 32.

[34] B. N. Roy and T. Wright, Cryst. Res. Technol. 31, 1039 (1996).

[35] H. Bross in: A. Seeger (Ed), Moderne Probleme der Metallphysik Vol 1, Springer-Verlag, Berlin 1965, p. 400.

[36] H.-U. Finzel, Bi Heping, and P. Wißmann, Z. Naturforsch. 52a, 640 (1997).

[37] K. L. Chopra, Thin Film Phenomena, Mc-Graw Hill 1969, p. 369.

[38] S. J. Soffer, J. Appl. Phys. 38, 1710 (1967).

[39] G. Thummes and H. H. Mende, Phys. Stat. Sol. (a) 26, 243 (1974).

[40] M. R. Pinnel, H. G. Tompkins, and D. E. Heath, Appl. Surf. Sci. 2, 558 (1979).

[41] V. Grajewski, H. H. Uchida, and E. Fromm, Thin Solid Films 193/194, 990 (1990). 